\title{
THE NUMBER OF Be STARS COMPARED WITH THE \\ NUMBER OF B STARS, TAKING INTO ACCOUNT THE \\ SPECIFIC PHYSICAL CHARACTERISTICS OF Be STARS
}

\author{
D. BRIOT \\ Observatoire de Paris, 61 Avenue de l'Observatoire, 75014 Paris \\ and \\ J. ZOREC \\ Institut d'Astrophysique de Paris, 98bis Boulevard Arago, 75014 Paris
}

It is very important for the understanding of the Be phenomenon, and particularly for locating a possible Be phase in the evolutionary track of $\mathrm{B}$ stars, to accurately determine the proportion of Be stars among all B stars. This type of study was already made several times in the past. Results obtained generally show a maximum Be frequency around spectral type B2 then a decrease towards late spectral types. Actually Be stars do not have the same characteristics as "normal" B stars and we have to take this into account in the determination of the ratio : number of Be stars / number of B stars. We use the Bright Star Catalogue (Hoffleit \& Jaschek 1982) and the Supplement to the Bright Star Catalogue (Hoffleit, Saladyga \& Wlasuk 1983) containing stars $V=7.10$ and brighter. This study needed to be made separately for the different spectral types because :

-Physical parameters of B stars are very different from B0 to B9 ;

-Emission characteristics of Be stars vary very much, with a decrease from B0e to B9e.

We successively consider three effects which can influence the frequency of Be stars :

- The over-luminosity of Be stars as compared with B stars ;

- Spectral type changes during constant mass evolution ;

- Spectral type changes due to the fast rotation of Be stars.

We know that Be stars are intrinsically brighter than B stars and more so when the stars are hotter (earlier type stars) (Zorec \& Briot 1991). If the stars are counted, as currently done, up to a limiting magnitude, Be stars are counted in a larger volume than B stars, which artificially increases the ratio of Be stars to B stars.

Stars change their spectral type while they evolve with a constant mass from the main sequence (luminosity class V) up to the giant branch (luminosity class III). The determination of the ratio of Be stars for the various spectral types must be done by classifying the stars of luminosity classes III and IV according to their mass, that is to say not according to their present 
spectral type but according to their spectral type they had when they were of luminosity class $\mathrm{V}$.

Because of the fast rotation of Be stars, we took into account the change of spectral type for the case of rigid rotation (Collins \& Sonneborm 1977). The distribution function of the angular velocity was calculated for each spectral type and luminosity class.

All results are shown on Fig. 1. We see that the ratios of Be stars to B stars change when specific physical characteristics of Be stars are taken into account. In particular, effects due to rotation shift the Be maximum towards early types. So we obtain the result that the hotter Be stars are, not only are the emission characteristics more pronounced, but also that the proportion of these stars is greater.

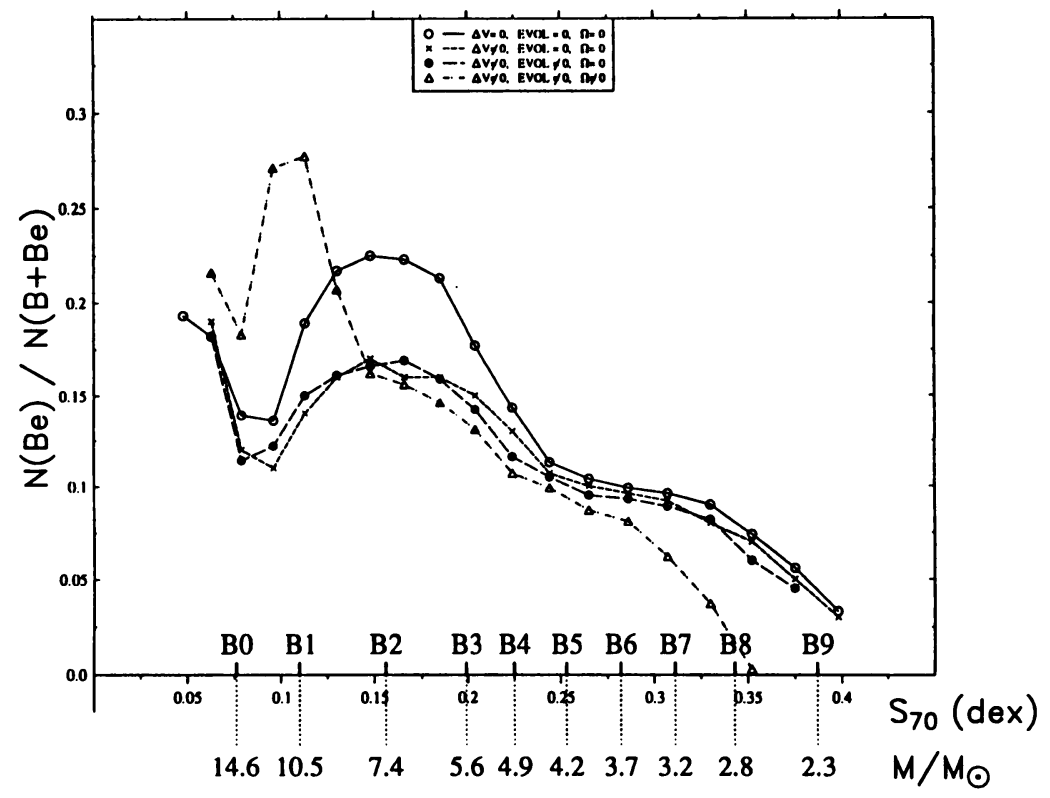

Fig. 1. Frequency of Be stars among B stars as a function of the spectral type. The results directly obtained from the Bright Star Catalogue and the Supplement to the Bright Star Catalogue are represented by open circles. The results are represented by crosses when the absolute magnitudes characteristic of Be stars are taken into account, by circled crosses when spectral type changes during stellar evolution are also taken into account and by triangles when spectral type changes due to fast rotation are in addition considered.

\section{References}

Collins, G.W., II and Sonneborn, G.H.: 1977, Astrophys. J. Suppl. Series 34, 41

Hoffleit, D. and Jaschek, C.: 1982, The Bright Star Catalogue, Yale Univ. Obs.

Hoffleit, D., Saladyga, M. and Wlasuk P.: 1983, A Supplement to the Bright Star Catalogue, Yale Univ. Obs.

Zorec, J. and Briot, D.: 1991, Astron.Astrophys. 245, 150 